\title{
Un criterio taxonómico para los patrones de consumo etílico
}

\author{
Taxonomic criterium for the alcohol consumption patterns
}

\author{
Ricardo González Menéndez \\ Profesor Titular de Psiquiatría. Facultad de Ciencias Médicas "Enrique Cabrera". La \\ Habana, Cuba.
}

\begin{abstract}
RESUMEN
Con el propósito de redactar un boceto sobre la taxonomía de los patrones de consume etílico para ser utilizado en los talleres nacionales de la oncena revisión de la Clasificación Internacional de Enfermedades, se proponen reflexiones sobre el concepto de bebedor social y las modalidades de consumo no social manifestadas tanto en personas con dependencia alcohólica como en otras sin dicho diagnóstico. A la luz de la trascendente resolución 58.26 se abordan los criterios de cantidad y frecuencia establecidos por la OMS, así como las razones cuantitativas que explican el mayor impacto del uso nocivo de alcohol en personas sin dependencia, que en los propios alcohólicos. Con objetivos taxonómicos, se propone excluir al consumo perjudicial de la categoría nosográfica alcoholismo y valorarlo como el escalón inmediato a la dependencia en el continuo de los patrones de ingestión que conduce a este estado. Destaca igualmente algunas modalidades de consumo de alta significación social cuando se valoran con criterios cuantitativos y cualitativos. Insiste en la metáfora de la decapitación prefrontal por el uso indebido de drogas duras, entre las que se incluyen el alcohol y la marihuana, por sus nefastos efectos sobre la conducta. Propone finalmente mayor atención a las manifestaciones somáticas derivadas de la ansiedad mantenida de los familiares convivientes con adictos a drogas duras en los que el estrés hace igual papel que el humo indirecto y se comportan como adictos pasivos cuya máxima expresión son los codependientes.
\end{abstract}

Palabras clave: Alcohol, patrones de consumo nocivo, reflexiones taxonómicas, adicción.

\section{ABSTRACT}


With the objective of making a draft on the taxonomy of alcohol consumption patterns for use in the national workshops of the $11^{\text {th }}$ Revision of the International Classification of Diseases, some reflections were submitted about the concept of social drinker and the non-social consumption modalities that manifest both in alcohol dependent-persons and non-dependent people. In the light of the transcendental resolution 58.26, the quantity and frequency criteria set by the WHO, as well as the quantitative reasons accounting for the higher impact of the harmful consumption of alcohol in non-dependent persons than in alcoholics were discussed. For taxonomic purposes, it was suggested that harmful consumption be excluded from the nosographic category alcoholism and be assessed as the immediate link to dependency in the patterns of drinking intake leading to this condition. Likewise, this paper underlined some consumption modalities of high social significance when they are evaluated based on quantitative and qualitative criteria. It insisted on the metaphor of frontal decapitation due to the use of hard drugs, including alcohol and marihuana, and their harmful effects on the behaviour. It finally made the proposal of paying more attention to somatic manifestations derived from sustained anxiety in relatives living with hard drug addicts, who are affected by the stress that plays the same role as the indirect smoke and behave as passive addicts whose ultimate expression is co-dependency.

Key words: Alcohol, harmful consumption patterns, taxonomic reflections, addiction.

\section{INTRODUCCIÓN}

En mayo de 2005, la resolución 58.26 de la OMS destacó en forma irrefutable, que los costos sociales determinados por el uso nocivo de alcohol son mayores que los determinados por los pacientes con dependencia alcohólica. ${ }^{1}$

Este documento de extraordinaria importancia conceptual y operativa para los programas integrales de atención a los problemas determinados por dicha droga modelo y portera, ratifica las observaciones de la mayoría de los adictólogos en el mundo no islámico. ${ }^{2}$

Dicho con otras palabras, en nuestros días la significación médico-social, económica y ético-humanística del uso indebido de bebidas alcohólicas en personas sin dependencia, es mayor que la determinada por quienes reúnen los criterios diagnósticos de dependencia al etanol, realidad estadística de fácil comprensión si se toman en cuenta los estimados actuales de que en la población mayor de 15 años, existen a nivel mundial de 10 a 12 consumidores de alcohol sin dependencia, por cada paciente con dicho diagnóstico. ${ }^{3}$

Es importante destacar que esta cifra de consumidores no dependientes, incluye tanto a quienes presentan un "consumo perjudicial" de esta droga (forma clínica temprana del alcoholismo según la Clasificación Internacional de Enfermedades Décima Revisión, equivalente al "abuso del alcohol" en el DSM-IV-R), como a otras personas con patrones de ingestión etílica ajenos a los del verdadero bebedor social y determinantes de nefastas consecuencias personales, familiares, escolares, laborales y comunitarias. ${ }^{4}$ 
Este último grupo de consumidores no alcohólicos, integra la mundialmente poco utilizada categoría de "personas bajo la influencia de alcohol", sustancia que pese a su condición legal, debía ser considerada como "droga dura", por su capacidad de bloquear funcionalmente la región prefrontal supraorbitaria y de liberar al sistema límbico con sus consecuentes conductas instintivo-afectivas, expresadas por impulsividad y violencia extrema, vinculadas a su consumo indebido en una alta proporción de supuestos bebedores sociales. ${ }^{5,6}$

Creemos que en la década que se aproxima y en el marco de la Oncena Revisión de la Clasificación Internacional de Enfermedades, resulta imprescindible establecer una nomenclatura actualizada que permita superar muchas denominaciones "acuñadas por la práctica", pero sin duda alguna, alejadas de la nitidez conceptual a que todos aspiran.

Como ejemplo de lo referido está el hecho de que el propio término de "uso nocivo" de alcohol destacado en la muy acertada resolución de la OMS puede superponerse sobre el de consumo perjudicial o abuso de alcohol y consideramos que la alerta de la OMS va más allá de esa categoría diagnóstica poco utilizada en la práctica asistencial por la habitual llegada tardía de los pacientes luego de pasar por las múltiples y ambiguas denominaciones clínico-epidemiológicas tales como consumo "social", moderado, heavy drinking, consumo indebido, consumo irresponsable, consumo inoportuno, consumo excesivo, consumo en bingo, consumo de riesgo, consumo problémico y consumo anormal. ${ }^{7}$

El propósito fundamental del presente trabajo es insistir en esta necesidad sentida, cuya satisfacción solo será lograda mediante consenso de especialistas nacionales y expertos internacionales durante la importante tarea taxonómica que se avecina. Por tanto, es importante invitar a reflexionar sobre el tema y exponer algunas apreciaciones sobre posibles modalidades de consumo que integran el uso nocivo de alcohol.

\section{EL PROBLEMA}

El primer tema a discutir podría ser si debe considerarse la categoría "uso nocivo" como equivalente a la primera etapa nosográfica del alcoholismo y nada más, o expandir dicho término para que incluya otros patrones de consumo etílico capaces de dañar al consumidor y sus convivientes en sus niveles biológico, psicológico, social, cultural y espiritual, potencialidades patogénicas generalmente presentes desde mucho tiempo antes de solicitar ayuda profesional.

El segundo propósito sería proponer para el debate algunas denominaciones para los patrones de consumo ajenos a los del verdadero bebedor social con la pretensión de cubrir las diferentes modalidades de comportamientos ante el alcohol.

Habida cuenta de la fácil valoración comunitaria y profesional del abstinente de por vida o después de una rehabilitación exitosa, el punto de partida en los debates debe ser la actualización del concepto de "verdadero" bebedor social, cuyo consumo debe carecer por definición, de consecuencias negativas sobre el sujeto o su entorno y que como demuestra la resolución 58.26 de la OMS requiere para su conceptualización trascender el énfasis en los clásicos parámetros de cantidad y frecuencia de consumo así como de estados de embriaguez ligeros, aceptados en su momento por la mayoría de los que nos dedicamos a la adictología. 
La generalización del consumo etílico solo fue posible tras el descubrimiento de la alfarería diez mil años atrás y a partir de entonces los comportamientos humanos ante dicha droga legal integraron tres grandes categorías: ${ }^{8}$

1. Los que se abstuvieron de su consumo en cantidad alguna.

2. Los que la consumieron en forma "realmente" social.

3. Los que hicieron un uso "no social" con dependencia o sin ella.

En los párrafos que vienen reflexionaremos sobre el segundo y tercer rubro de esta rudimentaria clasificación.

\section{Parámetros para identificar a un verdadero bebedor social}

El modelo clásico, para definir los bebedores sociales fue desarrollado en una etapa del enfrentamiento social al alcoholismo, en el que predominaba entre los científicos la preocupación por el efecto adictógeno del etanol en sus diferentes formas de presentación y se basó por tanto en el criterio hegemónico de cantidad y frecuencia del consumo. Se estableció así un "tope" de la ingestión etílica, "para prevenir los cambios metabólicos determinantes de la dependencia alcohólica."

La cifra de consumo no adictógeno se estableció por consenso de expertos como aquella en la que los preparados alcohólicos ingeridos no aportan más del $20 \%$ del total de calorías de la dieta del consumidor, lo que implica no sobrepasar el cuarto de botella de bebidas fuertes al $40 \%$ de alcohol, o su equivalente, una botella de vino al $10 \%$ o 5 latas de cerveza al $5 \%$, no más de tres veces en la semana. También se "aceptaba" hasta un estado de embriaguez ligera mensualmente.

Estos criterios propugnados por Marconi y aceptados por la mayoría de los especialistas -sobre todo para países con altas cifras de pacientes dependientes al alcohol- requieren a la luz de la resolución 58.26 una revalorización, pese a que por su objetividad indiscutible, el parámetro de cantidad y frecuencia se mantiene en los criterios actuales apoyados por la OMS, basados fundamentalmente en las tres primeras preguntas del cuestionario Alcohol use Disorder Identifcation Test (AUDIT), interrogantes que en forma sucesiva investigan la frecuencia de consumo, la cantidad consumida en un día normal y la frecuencia de episodios de consumo elevado. ${ }^{9}$

Sobre la base de considerar un trago como equivalente a $10 \mathrm{~g}$ de alcohol en Europa, (13 g en EE. UU.) y luego de señalar que cualquier nivel de consumo etílico implica peligros, se plantea por este prestigioso organismo mundial, que el consumo de riesgo es aquel que supera los $210 \mathrm{~g}$ de alcohol semanales en el hombre y los $140 \mathrm{~g}$ de alcohol en la mujer. Señala asimismo, que el tope que invierte los efectos del alcohol sobre algunas enfermedades en el sentido de prevenirlas o facilitarlas es de $10 \mathrm{~g}$ cada segundo día y que en dosis mayores, la curva en jota se desplaza hacia arriba como expresión de efectos perjudiciales.

Por otra parte, los actuales criterios de la OMS superan algunos planteamientos históricos de que las dos formas clásicas de alcoholismo (equivalentes al actual consumo perjudicial o abuso de alcohol y a la dependencia alcohólica) carecían de vínculos evolutivos y reconocen la existencia de un continuo que va desde la abstinencia a la dependencia, pasando por el consumo de bajo riesgo, el consumo de riesgo, el consumo perjudicial y la dependencia. Propugnan además, que la

http://scielo.sld.cu 
delimitación práctica de dichas etapas puede basarse en las tres primeras preguntas del cuestionario AUDIT instrumento de alto valor clínico epidemiológico. ${ }^{10}$

Se establecen así las siguientes categorías con algunas reflexiones del autor identificables mediante el subrayado.

- Consumo potencialmente beneficioso. Hasta $10 \mathrm{~g}$ de alcohol cada segundo día en el hombre o hasta $5 \mathrm{~g}$ de alcohol en la mujer.

- Consumo de bajo riesgo. Consumo regular diario de hasta $40 \mathrm{~g}$ de alcohol en el hombre o hasta $20 \mathrm{~g}$ de alcohol en la mujer y sin ningún día de ingestión en bingo.

- Consumo de riesgo. Consumo regular diario de 40 a $60 \mathrm{~g}$. de alcohol en el hombre o de 20 a $40 \mathrm{~g}$, en la mujer, expresa altas probabilidades de daños para la salud $\underline{0}$ afectación de la imagen familiar, laboral o comunitaria así como de su economía personal.

- Consumo perjudicial. Consumo regular diario de más de $60 \mathrm{~g}$ de alcohol en el hombre o más de $40 \mathrm{~g}$ en la mujer. Afecta tanto la salud física, como la mental.

- Dependencia del alcohol. Conjunto de fenómenos conductuales cognitivos y fisiológicos en los cuales el uso del alcohol se transforma en prioritario para el individuo, en contraposición a otras actividades y obligaciones que en algún momento tuvieron mayor valor para él. El deseo poderoso y difícil de controlar de consumir alcohol y la rápida reinstalación del cuadro al volver a beber después de un período de abstinencia, son también características centrales. Las expectativas evolutivas en quienes no se rehabilitan son la instalación o agravamiento de complicaciones somáticas y psíquicas así como el arribo a la fase final de vagabundeo con los paradigmas culturales del Skid Row o el clochard.

La OMS destaca las notables diferencias culturales en relación con la definición de bebida estándar y comenta que el factor 0,8 utilizado mundialmente para la valoración del contenido alcohólico, expresa que un $\mathrm{mL}$ de alcohol contiene $0,785 \mathrm{~g}$ de alcohol puro. ${ }^{7}$

Igualmente propone los siguientes valores para su definición a los que incorporamos los resultados de la formula (concentración alcohólica multiplicada por los cc del brebaje alcohólico a consumir y multiplicado además por el factor 0,8 para aproximarnos a los gramos contenidos en los mismos:

- $330 \mathrm{~mL}$ de cerveza al $5 \%$, una lata igual a $13,2 \mathrm{~g}$ de alcohol puro.

- $140 \mathrm{ml}$ de vinp al $12 \%$, un vaso igual a $13,4 \mathrm{~g}$.

- $165 \mathrm{~mL}$ de vino al $10 \%$, un vaso igual a $13,2 \mathrm{~g}$.

- $40 \mathrm{~mL}$ de bebidas espirituosas al $40 \%$, o una línea igual a $12,8 \mathrm{~g}$.

Es importante destacar, que en esta escala, las calorías vacías aportadas por el alcohol en quienes integran la categoría de consumo perjudicial, se corresponden o superan el $20 \%$ de las calorías totales de la dieta.

Si seleccionamos en el continente americano al sexo femenino por ser el más sensible y aplicamos la fórmula anterior, tendríamos de manera bastante aproximada que el tope que señala el paso a un consumo de riesgo sería no

http://scielo.sld.cu 
exceder el consumo regular de dos cervezas al $5 \%$ (o una cerveza fuerte), dos copas de vino al 10 o $12 \%$, o dos tragos de bebida fuerte por día, en tanto que el consumo beneficioso en el propio sexo valorado sería la cuarta parte y en días alternos, bastante más de la modalidad históricamente calificada como bebedor excepcional o discreto que al cabo de un año no sobrepasa las 5 ocasiones en que bebía como máximo una bebida estándar.

Ahora bien, de la misma manera que los estudios de terreno obligan al énfasis en parámetros cuantitativos, la valoración clínica será hegemónicamente cualitativa y se fundamenta en los siguientes criterios:

Criterio temporal. Más de dos meses de consistencia del patrón de consumo, preferimos valorar si dicho patrón está incorporado al estilo de vida.

Criterio de cantidad y frecuencia. Ya comentado.

Criterio de nocividad. Basado en la evidencia clínica de afectación biológica, psicológica o social.

Criterio de pérdida de libertad ante el alcohol. Falta de control cuando comienza el consumo, incapacidad para estar más de dos días en abstinencia o necesidad del alcohol para enfrentar actividades cotidianas.

Criterio de etiquetado. Con sus variantes de alo-etiquetado, apreciación de los demás acerca del patrón de consumo no social y auto-etiquetado que es la valoración del propio consumidor.

A la luz de la resolución 58.26 creemos oportuno reflexionar sobre la conveniencia de mantener la dependencia alcohólica como paradigma del alcoholismo y destacar como contrapartida la figura del consumo no social de las bebidas alcohólicas, categoría ajena a la abstinencia y al consumo realmente social.

Una posible definición del verdadero bebedor social tendría como matices cualitativos los siguientes:

- Cautela permanente ante el consumo de alcohol para evitar la embriaguez.

- Consumo preferentemente alimentario o en ocasiones especiales.

- Que el alcohol no "impregne" su estilo de vida ni dañe su economía.

- Consumo que no viole orientaciones médicas, ni normas culturales.

- Consumo que no afecte sus responsabilidades familiares, escolares, laborales o comunitarias.

Un marco de referencia práctico sería no sobrepasar las concentraciones de alcohol sanguíneo establecido en los diferentes países para poder conducir sin riesgo alguno un vehículo automotor, es decir, nunca más de 50 a 80 mg por 100 cc de sangre.

Como argumento fundamental para pensar en futuras modificaciones de la nomenclatura actual está el reconocimiento de que el verdadero consumo social de 
bebidas alcohólicas, sobre todo del vino tinto, tiende más a beneficiar la salud que a perjudicarla, debido a sus potencialidades como factor protector para diferentes afecciones y también la evidencia de que el uso no social deviene factor de riesgo de primerísimo orden, multiplicando en más de seis veces las probabilidades de instalación de las mismas. ${ }^{11}$

Cuando el consumo deja de ser realmente social y se hace irresponsable, inoportuno, con objetivos embriagantes, violatorio de orientaciones médicas como en madres gestando o lactando, enfermos mentales, epilépticos y menores de 18 años; cuando se consume en bingo o cuando el consumo embriagante se incorpora al estilo de vida, se delimitan en forma respectiva las diferentes modalidades de consumo no social, que pudieran ser:

1. Consumo irresponsable. Ejemplarizado por el conductor embriagado o por quien manipula en dicho estado una maquinaria peligrosa.

2. Consumo inoportuno. Cuyo prototipo es quien consume alcohol mientras presta algún servicio a la población, con altas probabilidades de desempeños inadecuados y riesgosos.

3. Consumo embriagante. Con los riesgos implícitos en el bloqueo prefrontal supraorbitario, metafóricamente denominado "decapitación prefrontal" al seguir el histórico modelo de Phineas Gage, el ferroviario inglés lesionado en dicha región cerebral.

4. Consumo contra criterios médicos. Con el catastrófico paradigma de la gestante que bebe o la madre que lacta, comportamientos cuyas cifras aún en países desarrollados supera el $12 \%$ de las mujeres en dichas situaciones.

5. Consumo en bingo. En hombres, cinco o más tragos por sesión de consumo en América (6 o más tragos en Europa). En mujeres, cuatro o más tragos por sesión de consumo, conductas manifestadas con mayor frecuencia en los fines de semana pero con potencialidades dismetabólicas inimaginables pese a estar científicamente bien demostradas.

6. Consumo de riesgo. En el que la embriaguez se incorpora al estilo de vida como antesala de las dos formas actuales de alcoholismo.

Por supuesto que estas modalidades de consumo no social pueden expresarse tanto en sujetos que cumplen los criterios diagnósticos actuales del alcoholismo en sus dos formas clínicas, como en quienes no los cumplen, con lo que se delimitarían dos subcategorías: los bebedores no sociales con dependencia alcohólica y los bebedores no sociales sin dicho diagnóstico.

Esta posibilidad taxonómica presenta empero el serio inconveniente de no ajustarse a la resolución 58.26 cuyo corte conceptual se establece entre el uso nocivo y la dependencia alcohólica.

La solución a este conflicto semántico pudiera estar en incorporar como variante del consumo no social al uso perjudicial o abuso de alcohol y que en vez de considerarlo una forma clínica de alcoholismo, fuese valorado como el paso previo e inmediato de la dependencia etílica.

De aceptarse este enfoque clasificatorio, la valoración comparativa de las formas de consumo no social que antes se comentaron, hace evidente que con la excepción

http://scielo.sld.cu 
del consumo contra criterios médicos madres gestando o lactando, pacientes psicóticos, epilépticos, o con cardio o hepatopatías y menores de 18 años, sub categoría que no requiere la embriaguez para determinar los efectos negativos, el resto del consumo no social tiene como esencia, la embriaguez alcohólica, condición que al "decapitar" temporalmente al sujeto en su región prefrontal supraorbitaria, hace retroceder 15 millones de años al ser humano en la evolución de las especies, toda vez que dicha región y sus trascendentes funciones no están presentes en ningún otro animal por desarrollado que este fuese.

Estos efectos del alcohol -comunes a todas las drogas duras a partir de sus efectos transformadores de la conciencia, la personalidad y el comportamiento- se expresan por la trágica afectación de las virtudes, principios y normas éticomorales, al bloquearse el freno de las conductas instintivo afectivas, acción que junto a la liberación de los centros subcorticales -sobre todo límbicos- hace que el sujeto se comporte -en lenguaje freudiano- como carente de SUPER YO; con evidente debilidad del YO; y como dotado de un ELLO brutalmente hegemónico.

Se explica así la existencia de la incertidumbre ante el ¿cómo vendrá? de los convivientes y también lo escuchado a un paciente alcohólico años atrás: "cuando llego embriagado a mi casa, hasta mi perro se esconde bajo la cama."

Cuando se calculan los costos sociales del uso no social de drogas en países desarrollados como EE. UU. y se contabilizan los gastos médicos, policiales, jurídicos, de seguros, carcelarios, la disminución de la productividad del trabajo y de la producción de bienes, así como los daños a la propiedad y los implícitos en la extracción pasiva de liquidez, se aprecia que las drogas que denominamos blandas - por no afectar en forma relevante la conducta- lideradas por el tabaco, determinan el $50 \%$ anual de dicha repercusión, de 548 mil millones de USD, en tanto que el alcohol se aproxima al $27 \%$ y el resto de las drogas al $23 \%$, bien entendido que los estimados referidos no toman en cuenta la repercusión médico social y económica de la criminalidad vinculada a las sustancias ilícitas. ${ }^{12}$

El uso no social de alcohol, manifestado por los dependientes a dicha droga y el resto de la población mayor de 15 años, con la excepción de los abstemios y los "verdaderos bebedores sociales", determina cada año a nivel mundial: 3,2 \% de todos los fallecimientos, 12 años de reducción promedio de la esperanza de vida y 4 $\%$ del total de años de vida productiva perdidos por discapacidades y muertes precoces y estas cifras son aún mayores en nuestro continente.

En la región de Las Américas, el uso no social de alcohol es el más importante factor de riesgo para enfermar entre los 27 monitoreados por OPS/OMS y supera entre otros a la presión arterial elevada, el tabaquismo, la obesidad y la vida sedentaria. ${ }^{13}$

De hecho este uso no social de alcohol se vincula a más de sesenta enfermedades que incluyen afecciones tan poco esperadas como el cáncer de mama y la osteoporosis sobre todo masculina. ${ }^{14}$

Es igualmente importante destacar que la repercusión del uso no social de alcohol y del consumo del resto de las drogas duras sobre otras personas, no se limita a los accidentes o lesiones infligidas de manera intencional o no, ni al incesto, violaciones, trastornos de estrés postraumático, ansiedad, depresión y suicidios, sino que trasciende con mucho dichos efectos para determinar también discapacidades y muertes sobre los convivientes, al reproducir el patrón descrito en los fumadores pasivos, con la diferencia de que ahora el agente agresor no es el humo indirecto, sino la ansiedad mantenida, que al expresarse mediante las vías

http://scielo.sld.cu 
vegetativas, endocrino-metabólicas y cráneo-espinales determina hipertensión, úlceras gastroduodenales, arritmias cardíacas, obesidad, hiperlipidemias, accidentes trombóticos coronarios y encefálicos, hipertiroidismo, diabetes, alopecia, liquen plano y psoriasis, secuelas que a veces olvidamos y que constituyen formas ocultas de lo que proponemos llamar alo morbilidad, alo comorbilidad y alo mortalidad, figuras clínico epidemiológicas generalmente subvaloradas y ocasionalmente ausentes entre nuestras expectativas de efectos nocivos de las drogas duras. ${ }^{15}$

Desconocer estas posibilidades nosográficas determinadas por el estrés mantenido y su derivación, implicaría negar la existencia del eje psico-neuro-inmuno-hipófisoadreno-córtico-gonadal, que constituye la esencia de los cuadros antes llamadas psicosomáticos, corticoviscerales o psicofisiopatológicos y hoy incluidos en la Décima revisión de la Clasificación Internacional de Enfermedades en su capítulo $\mathrm{V}$ bajo el rubro diagnóstico de F45 Trastornos somatomorfos y F54 Trastornos psicológicos y del comportamiento en trastornos o enfermedades clasificados en otro lugar. ${ }^{4,16}$

Estas realidades clínico-epidemiológicas plantean igualmente la necesidad de llegar a consenso sobre la denominación de quienes conviven con los consumidores no sociales y cuyo nivel de ansiedad alcanza su clímax en quienes conviven con dependientes a drogas duras y que por mecanismos de negación rechazan por mucho tiempo la ayuda rehabilitatoria.

Estas personas víctimas de un paradigma patogénico cercano al fumador "pasivo" podrían denominarse en forma general como "adictos" pasivos y con mayor especificidad como alcohólicos "pasivos", marihuaneros "pasivos", cocainómanos "pasivos", ludópatas "pasivos" y otros términos que expresen en forma simultánea, la variante de adicción del consumidor y el papel de víctima del conviviente. Cuando dicha situación llega al clímax y las preocupaciones sobre el consumo del "ser querido" así como las formas de pretender evitarlo devienen tema central de la existencia, podríamos utilizar -con especial selectividad por el alto riesgo de enfermar- la acertada denominación de codependiente, Estas son las esposas y madres que en horas de la madrugada esperan en vigilia el regreso del paciente alcohólico o del adicto a otras drogas duras, con la gran incertidumbre del ¿cómo vendrá?, la misma que seguramente hubiese restado años de vida a la progenitora de Juan Cristóbal, el protagonista de la joya literaria de Romain Rolland, en la que Luisa su madre, esperaba presa de angustia el retorno de Melchor, su esposo.

Cuando la ciencia demuestre la trascendencia médico-social y humanística de estas expresiones somáticas que reducen la calidad y expectativa de vida de los convivientes, el iceberg de las drogas se hará aún más monstruoso.

Y qué decir de esa gran desgracia familiar llamada en sus diferentes expresiones como "síndrome del feto" alcohólico o como bien destaca el querido maestro Don Francisco Alonso Fernández, síndrome embriofetal alcohólico, conocido desde el Siglo XVI cuando en plena "epidemia de la ginebra" en Inglaterra, se hicieron evidentes los efectos teratogénicos morfológicos del alcohol consumido por las gestantes, para más tarde identificarse en el ámbito especializado que, además de las anormalidades corporales, los efectos transplacentarios del alcohol determinan retraso mental así como teratogenias conductuales expresadas por temperamentos proclives a la impulsividad, explosividad, intolerancia a frustraciones, incapacidad para posponer satisfacciones, labilidad afectiva e hiperquinesia; características que constituyen terreno abonado para la instalación de personalidades disociales y en el mejor de los casos, de trastornos de atención e hiperquinesia, afecciones que facilitan la instalación de adicciones a drogas duras. ${ }^{17}$ 
En el siguiente figura pretendemos correlacionar las modalidades de comportamientos ante el alcohol y destacar la repercusión global del uso no social que incluye a muchos supuestos bebedores sociales.

\section{I-Abstemios}

II-Bebedores sociales

III-Bebedores no sociales sin dependencia

III.1. Consumo irresponsable

III. 2. Consumo inoportuno

III.3. Consumo embriagante

III.4. Consumo contra criterios médicos

III.5. Consumo en bingo

III.6. Consumo de riesgo

III.7. Consumo perjudicial

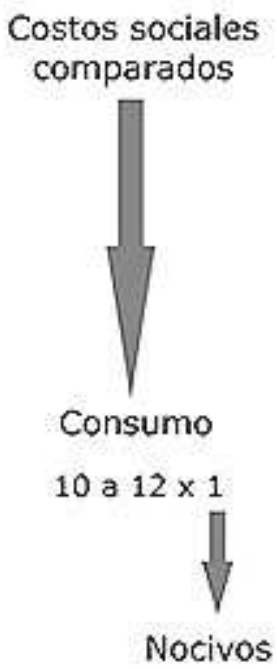

Pacientes alcohólicos

IV.Bebedores no sociales con dependencia (iguales patrones de III.1 a III.7)

(el alcohol como interés central)

Nocivos

IV.1. Bebedores no sociales con dependencia y complicaciones

IV.2. Bebedores no sociales con dependencia y en fase final

Fig. Comportamientos ante el alcohol y costos sociales (resolución 58.26 OMS).

Finalmente se puede concluir que a punto de partida del contenido del la resolución 58.26 de la OMS que destaca la mayor relevancia social del consumo nocivo de alcohol que el de los pacientes con dependencia alcohólica ante la expectativa de los talleres internacionales para la oncena revisión de la Clasificación Internacional de Enfermedades, consideramos que el significado del término "consumo nocivo" abarca muchas modalidades de consumo que trascienden los limites del verdadero bebedor social y se manifiestan tanto en presuntos bebedores sociales, como en los dependientes al alcohol. Estas proposiciones serán debatidas como boceto en los talleres a efectuarse en Cuba en fecha próxima.

\section{REFERENCIAS BIBLIOGRÁFICAS}

1. Organización Mundial de la Salud. Resolución 58.26 de la 58 Asamblea Mundial de la salud "Problemas de salud pública, causados por el uso nocivo del alcohol". Ginebra: OMS; 2005.

2. González R, Galán G. La declaración de Brasilia. Reflexiones sobre sus pautas para el enfrentamiento a los problemas relacionados con el alcohol. Rev Hosp Psiq Habana [serie en Internet]. 2007 [citado 29 Sep 2010];4(1). Disponible en: http://www.revistahph.sld.cu/

3. González R, Galán G. El alcoholismo: reflexiones sobre tres décadas de investigación en el Hospital Psiquiátrico de La Habana. Rev Hosp Psiq Habana [serie en Internet]. 2007 [citado 29 Sep 2010];4(2). Disponible en:

http://www.revistahph.sld.cu/ 
4. Otero A. Tercer Glosario Cubano de Psiquiatría. Glosario Cubano de la Décima Revisión de la Clasificación Internacional de Enfermedades, Capítulo V. GC-3. Hospital Psiquiátrico de La Habana; 2008.

5. Hurd Y. Perspectiives on Current Directions in the Neurobiology of addiction. Disorders Relevant to Genetic Risk Factors. CNS Spectrums. 2006;11(11):855-62.

6. Hoppes K. The Application of Mindfulness Based Cogniitive Interventions in the Treatment of Co-occurring Addictive and Mood Disorders. CNS Spectrums. $2006 ; 11(11): 829-51$.

7. Anderson P, Gual A, Colón J. Alcohol y atención primaria de la salud. Informaciones clínicas básicas para la identificación y el manejo de riesgos y problemas. Washington. D.C.: OPS; 2008.

8. González R. Las adicciones a la luz de la ciencia y el símil. Caracas: Editorial Fundación José Félix Ribas; 2008.

9. Johnson B, Ruiz P, Galanter M. Handbook of Clinical Alcoholism treatment. Baltimore, Lippincott: Williams and Wilkins; 2004.

10. Babor T, Higgins-Biddle, Saunders J, Monteiro M. Cuestionario de identificación de trastornos debidos al consumo de alcohol. Ginebra: OMS; 2001.

11. World Health Organization. Global Status Report on Alcohol. Geneva: WHO; 2004.

12. Kaplan H, Sadock B. Synopsis of Psychiatry: Behavioral Sciences/Clinical Psychiatry. 9na ed. Baltimore: Williams and Wilkins; 2004.

13. Roses Periago M. La Salud Mental; una prioridad de Salud Pública en las Americas. Rev Panamer Salud. 2005;18(94-5):223-5.

14. Rehm J, Monteiro M. Alcohol consumption and burden of diseases in the Americas. Implications for Alcohol Policy. Rev Panamer Salud. 2005;18(94-5):2418.

15. González R. Significación médico social y ético humanística de las drogas. Rev Cubana Salud Pública [serie en Internet]. 2007 [citado 29 Sep 2010];33(1).

Disponible en: http://www.scielosp.org/scielo.php?pid=S0864$\underline{34662007000100002 \& \text { script }=\text { sci arttext\&tlng }=e n}$

16. Arce S. Inmunología Clínica y Estrés. La Habana: Editorial Ciencias Médicas; 2007.

17. Alonso Fernández F. Los secretos del alcoholismo. Mujer, trabajo, juventud. Madrid: Ediciones Libertarias; 1998.

Recibido: 16 de febrero de 2010.

Aprobado: 30 de julio de 2010. 
Ricardo González Menéndez. Jefe del Departamento de Atención a Adicciones del Hospital Psiquiátrico de La Habana "Dr. Eduardo Ordaz". Facultad de Ciencias Médicas "Enrique Cabrera". La Habana, Cuba. Correo electrónico:

isaric@infomed.sld.cu 